\title{
In-plane commensurate GaN/AIN junctions: Single-layer composite structures, single and multiple quantum wells and quantum dots
}

\author{
A. Onen, ${ }^{1}$ D. Kecik, ${ }^{1}$ E. Durgun,,${ }^{1, *}$ and S. Ciraci ${ }^{2, \dagger}$ \\ ${ }^{1}$ UNAM-Institute of Materials Science and Nanotechnology, Bilkent University, 06800 Ankara, Turkey \\ ${ }^{2}$ Department of Physics, Bilkent University, 06800 Ankara, Turkey \\ (Received 3 December 2016; revised manuscript received 2 March 2017; published 21 April 2017)
}

\begin{abstract}
In-plane composite structures constructed of the stripes or core/shells of single-layer GaN and AlN, which are joined commensurately, display a diversity of electronic properties that can be tuned by the size of their constituents. In heterostructures, the dimensionality of the electrons changes from two dimensional (2D) to one dimensional (1D) upon their confinements in wide constituent stripes, leading to the type-I band alignment and hence multiple quantum well structure in the direct space. The $\delta$ doping of one wide stripe by another narrow stripe results in local narrowing or widening of the band gap. A single quantum well structure is acquired from the finite-size AlN-GaN-AlN junctions. In a patterned array of GaN/AlN core/shells, the dimensionality of the electronic states is reduced from two dimensional to zero dimensional, forming multiple quantum dots in large GaN cores, while 2D electrons propagate in multiply connected AIN shell as if they are in a supercrystal. A consistent and detailed discussion of the effects of confinement in momentum and direct spaces is provided. As a result of confinement, the variation of the band gap in the direct space is found to be rather different from the edges of the conduction and valence bands inferred from the band edges of constituent 2D single-layer GaN and AlN. Even if all the results in this study pertain to the free-standing single-layer composite structures, the effects of the different substrates over which these composites can grow are examined in detail. This study unveils the potential of composite structures in designing novel nanomaterials. These predictions are obtained from first-principles calculations based on density functional theory on 2D GaN and AlN compound semiconductors which were synthesized recently.
\end{abstract}

DOI: 10.1103/PhysRevB.95.155435

\section{INTRODUCTION}

In the past, commensurate three-dimensional (3D) heterostructures of III-V compounds such as AlAs/GaAs (or ALGAS) and GaN/AIN have dominated the research on the physics of electrons in lower dimensionality displaying fundamental features and novel quantizations with potential electronic device applications [1-6]. Following the syntheses of single-layer (SL) graphene, $h$-BN and $h-\mathrm{MoS}_{2}$, the question of whether in-plane heterostructures of two-dimensional (2D) nanomaterials can be realized has been frequently asked [7-9]. Already in 2008, novel features of in-plane composite structures or heterostructures of nanoribbons of BN/graphene [10], GaN/AlN [11], semiconducting graphene nanoribbons with graded width forming multiple quantum well structures [12], and recently $\mathrm{GaN} / \mathrm{SiC}$ heterostructures [13] were revealed theoretically. Even the nanowires of silicon with graded cross section [14] and carbon nanotubes [15] were shown to develop complex quantum structures with interesting resonance tunneling effects. The fabrication of BN/graphene lateral heterostructures has been taken as an important step towards the development of atomically thin integrated circuitry with electrically active and passive elements embedded in continuous sheets [16-20]. Most recently, atomically sharp inplane heterostructures of $\mathrm{WS}_{2} / \mathrm{MoS}_{2}$, which generate localized photoluminescence enhancement and a $p$ - $n$ junction behavior have been already fabricated [21]. More recently, unusual features of in-plane (graphene $)_{p} /(\mathrm{BN})_{q}$, metal/insulator com-

\footnotetext{
*durgun@unam.bilkent.edu.tr

†ciraci@fen.bilkent.edu.tr
}

posite structures [22], and graphene/BN/graphene in-plane nanocapacitors [23] were predicted.

SL 2D GaN and AlN nanostructures are essential ingredients for the realization of GaN/AIN in-plane heterostructures, i.e., an analog of ALGAS type junctions in an atomic plane. As early as 2005 [24], specific III-V compound semiconductors have been predicted that they can form stable, single-layer and multilayer structures with exceptional electronic properties [11,25-27]. In particular, SL 2D GaN and AlN compounds have been predicted to have planar honeycomb structures since the anion $\mathrm{N}$ from the first row of the periodic table strengthens $\pi-\pi$ bonds in order to maintain the planar geometry $[11,24]$. They are nearly lattice matched and have wide fundamental band gaps of different widths. These findings based on firstprinciples calculations herald that the in-plane, commensurate growth of SL GaN and AlN could be realized to fabricate the composite structure or heterostructures with a diversity of optoelectronic properties. Fortunately, earlier theoretical predictions come to reality; very thin layers of AlN have been synthesized [28]. More recently, 2D GaN has been synthesized via graphene encapsulation, an important achievement which will render $\mathrm{GaN}$ an important nanomaterial in 2D flexible optoelectronics [29].

In this paper, we show that stable, in-plane composite materials $(\mathrm{GaN})_{p} /(\mathrm{AlN})_{q}$, or simply $(p / q)$, can be constructed of periodically repeating stripes of $\mathrm{GaN}$ and $\mathrm{AlN}$ continuously (or commensurately) joined along their zigzag $(Z)$ edges. Here, $p$ and $q$, which indicate the numbers of formula units of the constituent compounds in the unit cell, are controllable parameters. These composite materials exhibit a wide range of physical properties depending on the values of $p$ and $q$ : A structure having $p \simeq 1-2$, but very large $q$, or vice versa, 
is identified as a $\delta$ doping, where very narrow ribbon of $\mathrm{GaN}$ is implemented commensurately in a large AlN stripe, or vice versa. For $p=1-2$, and $q=1-2$, the composite structure behaves differently from its parent constituents as if a compound of lines of GaN and AlN. For small $p$ and $q$, the fundamental band gap of the composite can be tuned by varying $p$ and $q$. On the other hand, for large $p$ and $q$, the electronic states become confined to one of the constituent stripes, experiencing a transition from $2 \mathrm{D}$ to one-dimensional (1D). At the boundary region of the junction, charge is transferred from one region to another and normally sets the band lineup, constructing multiple quantum wells and quasi-one-dimensional (quasi-1D) quantum structures in a $2 \mathrm{D}$ atomically thin nanomaterial with diverse functionalities.

The dimensionality can be further lowered from 1D to zero dimensional (0D) by constructing patterned GaN-core and AlN-shell structure or quantum dots. While the present analysis uses periodic systems in $2 \mathrm{D}$, composite structures can also be constructed from the combination of finite number of lattice-matched stripes or core/shell structures forming a mesh. For example, the junction of the stripes AlN-GaN-AlN can construct a single quantum well. The level spacing of confined states can be tuned by the number of formula unit $p$. Since both constituents have been synthesized recently, novel composite materials constructed thereof hold a great promise in optoelectronics, which can be exploited in the rapidly developing field of $2 \mathrm{D}$ electronics. The predictions of this study are obtained from first-principles calculations based on density functional theory (DFT), detailed in the next section. Many features revealed in this paper can find validity in lattice-matched, in-plane composite structures of other IIIV, II-VI compounds, transition-metal dichalcogenides/oxides, pseudomorphic group IV-IV elements, and pnictogens.

\section{METHOD}

The ground-state total energy and electronic structure calculations of $2 \mathrm{D}$, in-plane $\mathrm{SL}(p / q)$ composites have been performed within spin-polarized DFT. The projectoraugmented-wave potentials (PAW) formalism [30,31] is used as implemented in the Vienna $a b$ initio simulation package (VASP) [32,33]. The electron exchange and correlation (XC) potential is described by the Perdew-Burke-Ernzerhof (PBE) form within the generalized gradient approximation (GGA) with $d$ electrons also taken into account (GGA- $d \mathrm{XC}$ potential) [34]. An exception to using the standard PBE XC functional has been for the total and Bader charge density calculations of the $\mathrm{Al}$ atom, since it was necessary to include the contribution of $2 s 2 p$ orbitals to the ground-state charge. The plane-wave basis set was used with an energy cutoff of $520 \mathrm{eV}$ for all calculations. The van der Waals interactions were considered for all 2D in-plane heterostructure systems [35,36]. The conjugate gradient method was employed for optimizing the atomic positions. The total energy and atomic forces were minimized with an energy difference between the sequential steps set to $10^{-5} \mathrm{eV}$ for convergence. The maximum allowed force on each atom and Gaussian smearing factor were taken as $0.05 \mathrm{eV} / \AA$ and $0.05 \mathrm{eV}$, respectively. As for the Brillouin zone (BZ) sampling [37], $\Gamma$-centered $26 \times 26 \times 1 \mathbf{k}$ grid was undertaken for the rectangular unit cell of GaN (AlN) and scaled accordingly for larger systems. In order to avoid the spurious interactions between the periodic images, a supercell with $\sim 15 \AA$ vacuum space was adopted.

The interatomic charge transfer between Ga-N and Al-N pairs (bonds) was carried out for the zigzag $p=14$ and $q=14$ heterostructures, using the Bader charge analysis method, by increasing the criterion for the precision of the calculations [38].

$A b$ initio, finite-temperature molecular dynamics (MD) calculations have been performed for $Z:(2 / 2)$ composite structure using a $(2 \times 4)$ supercell. A microcanonical ensemble is simulated by scaling velocities at each 1 -fs time step.

\section{2D SINGLE-LAYER GAN AND ALN}

The optimized atomic structure, calculated electronic energy bands together with the total and atom projected densities of states and optical spectra of the parent constituents, namely 2D GaN and AlN, are presented in Fig. 1 as reference for the forthcoming discussions. Both of them are semiconductors with indirect band gaps of 2.16 and $2.91 \mathrm{eV}$, respectively. Results of the previous calculations regarding the energy band gaps of GaN and AlN and their extensive discussions can be found in Refs. [25-27]. Recent calculations predict that the exciton binding energies of GaN and AlN are substantially strong [27,39] giving rise to excitonic peaks well below the band gap, revealing binding energies of nearly 1.2 and $1.7 \mathrm{eV}$, respectively. The density of states at the bottom of the conduction band is low, but the flat bands at about $5 \mathrm{eV}$ give rise to sharp peaks, which contribute to high absorption in this energy range. The cohesive and formation energy of SL GaN (AlN) at $T=0 \mathrm{~K}$ are 8.04 and $-0.71 \mathrm{eV}(10.43$ and $-1.02 \mathrm{eV})$. Based on these energy considerations and $a b$ initio phonon and molecular dynamics calculations, both free-standing SL GaN and AlN structures are stable and correspond to local minima in the Born-Oppenheimer surface. Their lattice constants in 2D rectangular cell differ only by $2.5 \%$. They also share the same anion $\mathrm{N}$, attributing them a planar geometry.

\section{GaN/AIN IN-PLANE COMPOSITE STRUCTURES}

The atomistic model to study the in-plane composite structures of GaN and AlN joined commensurately along their zigzag edges, $Z:(\mathrm{GaN})_{p} /(\mathrm{AlN})_{q}$ or shortly $Z:(p / q)$, is presented in Fig. 2. Here, $p$ and $q$ are the number of formula units of $p$ and $q$ of $\mathrm{GaN}$ and AlN, respectively, in the unit cell. These are the parameters which can directly control the physical properties discussed in forthcoming sections. GaN and AlN domains are separated by two different sharp boundaries (or interfaces), namely, $\mathrm{I}_{1}$ and $\mathrm{I}_{2}$. Interfaces of the bulk heterostructures formed by $3 \mathrm{D} \mathrm{GaN}$ and $\mathrm{AlN}$ crystals have been produced with the accuracy of a monolayer. Already, in-plane $\mathrm{MoS}_{2} / \mathrm{WS}_{2}$ heterostructures with atomically sharp boundaries have been fabricated [21]. Structure optimizations, MD simulations in this study, energetics related with the formation and growth discussed in the next section present convincing evidences that GaN/AlN junctions can be realized with atomically sharp boundaries. 

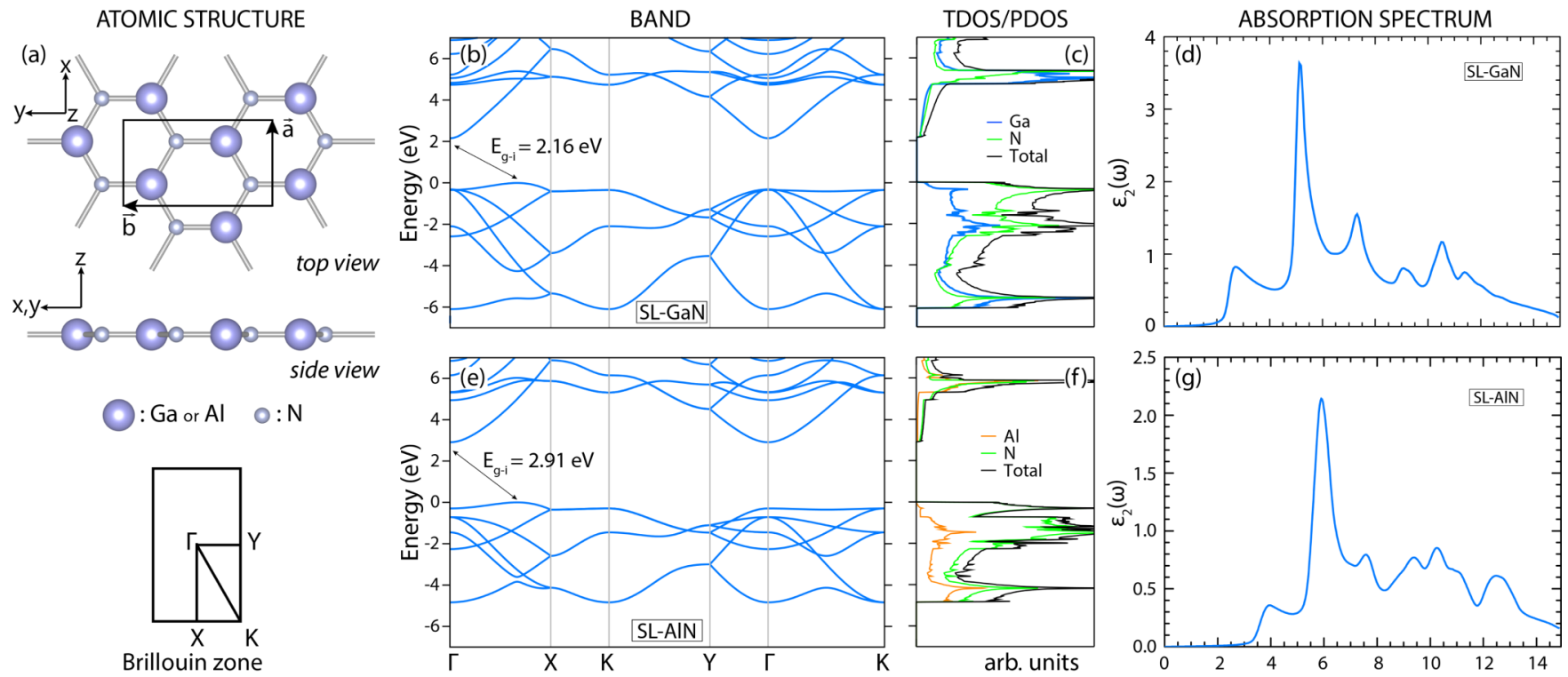

FIG. 1. (a) Top and side views of the atomic structure of constituent 2D SL GaN and AlN together with the lattice vectors $\mathbf{a}$ and $\mathbf{b}$ of the rectangular cell. (b) The electronic energy band structure of SL GaN along the high-symmetry directions of the Brillouin zone corresponding to the rectangular cell. (c) Corresponding total and atom projected densities of states, TDOS and PDOS. (d) Optical absorption spectra. (e)-(g) Same for SL AlN. The zero of energy is set at the top of the valence bands. The indirect band gaps $E_{g, i}$ are indicated by arrows.

\section{A. Energetics and stability}

The energetics of the in-plane composite structures can be characterized by their average cohesive, formation, and junction energies. The average cohesive energies of $2 \mathrm{D}$ in-plane $Z:(p / q)$ relative to the constituent atoms are calculated from the expression $\bar{E}_{c}=\left\{p \times E_{T}[\mathrm{Ga}]+q \times\right.$ $\left.E_{T}[\mathrm{Al}]+(p+q) \times E_{T}[\mathrm{~N}]-E_{T}[(p / q)]\right\} /(p+q)$ in terms of the total energies of free $\mathrm{Ga}, \mathrm{Al}, \mathrm{N}$ atoms and that of $Z:(p / q)$. The higher the positive $\bar{E}_{c}$, the stronger is the binding. The average formation energy $\bar{E}_{f}[(p / q)]=$ $E_{c}[(p / q)]-\left(p \times E_{c}[3 \mathrm{D} \mathrm{GaN}]+q \times E_{c}[3 \mathrm{D} \mathrm{AlN}]\right) /(p+q)$ is calculated relative to the $3 \mathrm{D}$ global minima by subtracting the cohesive energies of 3D bulk GaN and AlN crystals from the average cohesive energy of the composite

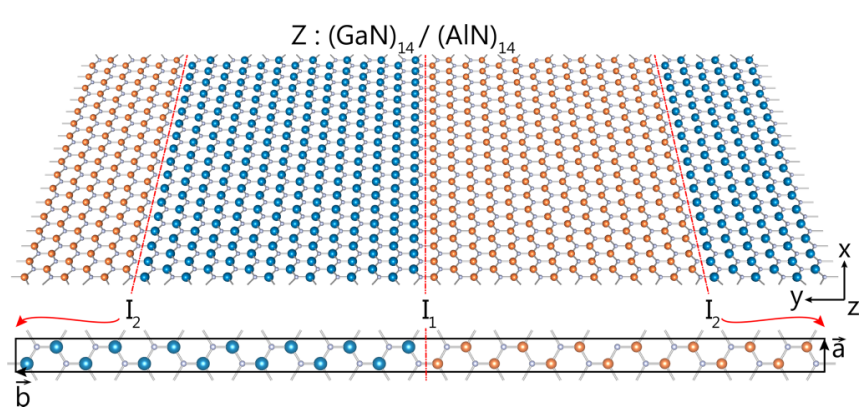

FIG. 2. Perspective view of the atomic structure of $Z:(\mathrm{GaN})_{p} /(\mathrm{AlN})_{q} \quad$ or $Z:(p / q)$ with $\left.p=q=14\right]$, which is formed by the stripes of SL GaN and AlN joined commensurately along their zigzag $(Z)$ edges. The unit cell together with the fundamental lattice vectors $\mathbf{a}$ and $\mathbf{b}$ and two different boundaries between $\mathrm{GaN}$ and $\mathrm{AlN}, \mathrm{I}_{1}$ and $\mathrm{I}_{2}$, are also shown. $\mathrm{Ga}, \mathrm{Al}$, and $\mathrm{N}$ atoms are represented by large turquoise, medium orange, and small gray balls, respectively. structure. The average formation energy of a junction relative to $2 \mathrm{D} \mathrm{SL}$ constituents is defined as $\bar{E}_{j}[(p / q)]=$ $p \times E_{T}[2 \mathrm{D} \mathrm{GaN}]+q \times E_{T}[2 \mathrm{D} \mathrm{AlN}]-E_{T}[(p / q)]$ and are calculated in terms of the total energies of $2 \mathrm{D} \mathrm{SL}$ GaN (per formula unit), i.e., $E_{T}[2 \mathrm{D} \mathrm{GaN}]$ and of $2 \mathrm{D} \mathrm{SL} \mathrm{AlN}$, $E_{T}[2 \mathrm{D} \mathrm{AlN}]$, and the total energy of $Z:(p / q), E_{T}[(p / q)]$ (per unit cell). Accordingly, if $\bar{E}_{j}>0$, the junction formation is favored. In Table $\mathrm{I}$, the optimized lattice constants, $\bar{E}_{c}, \bar{E}_{f}, \bar{E}_{j}$ energies, and the fundamental band gaps of composite structures are given as a function of $p$ and $q$. Calculated values of 3D (wurtzite) and 2D honeycomb structures of the constituents are also included for the sake of comparison.

While the calculated average cohesive energies of $Z:(p / q)$ are all positive relative to free $\mathrm{Ga}, \mathrm{Al}$, and $\mathrm{N}$ atoms and favors binding, their formations at $T=0 \mathrm{~K}$ relative to their parent $3 \mathrm{D}$ crystals in wurtzite structure have small negative energies. This indicates that the formation of $Z:(p / q)$ is unfavorable relative to the parent $3 \mathrm{D} \mathrm{GaN}$ and AlN crystals in wurtzite structure. These results suggest that the $Z:(p / q)$ composites may correspond to local minima on the Born-Oppenheimer surface. Whether the composite structures are in deep minima and remain stable against thermal excitation require stability analysis based on ab initio finite-temperature MD simulations. We performed these tests as detailed in the Method section. We considered $Z:(2 / 2)$, which is one of the composites most likely to be prone to thermal instabilities and carried out MD simulations for $0.5 \mathrm{ps}$ at temperatures 200, 400, 600, and $800 \mathrm{~K}$. Finally, at $1000 \mathrm{~K}$, we performed MD simulations for 2 ps. The snapshots of the atomic configurations of the $(2 \times 4)$ supercell at the end of $0.5 \mathrm{ps}$ simulation time performed at a given temperature are shown in Fig. 3. As temperatures are raised, the displacements of the atoms from their equilibrium positions increase. However, even at $1000 \mathrm{~K}$, the honeycomb structure of the composite is found to be robust and stable. 
TABLE I. 3D and 2D constituents and their composite structures specified by $Z:(p / q)$; lattice constants $a$ and $b$; cohesive energy $\bar{E}_{c}$ averaged per $\mathrm{Ga}(\mathrm{Al})-\mathrm{N}$ pair; formation energy $\bar{E}_{f}$ at $T=0 \mathrm{~K}$ averaged per $\mathrm{Ga}(\mathrm{Al})$-N pair; junction formation energy $\bar{E}_{j}$ per unit cell; the fundamental band gap $(d)$ direct or $(i)$ indirect.

\begin{tabular}{lccccr}
\hline \hline $\begin{array}{l}\text { Composite } \\
(p / q)\end{array}$ & $\begin{array}{c}a \\
(\AA)\end{array}$ & $\begin{array}{c}b \\
(\AA)\end{array}$ & $\begin{array}{c}\bar{E}_{c} \\
{[\mathrm{eV} / \mathrm{Ga}(\mathrm{Al})-\mathrm{N}]}\end{array}$ & $\begin{array}{c}\bar{E}_{f} \\
{[\mathrm{eV} / \mathrm{Ga}(\mathrm{Al})-\mathrm{N}]}\end{array}$ & $\begin{array}{c}\bar{E}_{j} \\
(\mathrm{eV})\end{array}$ \\
\hline $3 \mathrm{D} \mathrm{GaN}$ & 3.22 & 3.22 & 8.76 & & $\begin{array}{c}E_{g, d} / E_{g, i} \\
(\mathrm{eV})\end{array}$ \\
$3 \mathrm{D} \mathrm{AlN}$ & 3.13 & 3.13 & 11.45 & & $4.71(d)$ \\
$2 \mathrm{D} \mathrm{GaN}$ & 3.21 & 3.21 & 8.04 & -0.71 & $2.06(d)$ \\
$2 \mathrm{D} \mathrm{AlN}$ & 3.13 & 3.13 & 10.43 & -1.02 & $2.91(i)$ \\
$Z: 1 / 1$ & 3.17 & 5.49 & 9.23 & -0.87 & $2.62(i)$ \\
$Z: 2 / 2$ & 3.17 & 10.98 & 9.23 & -0.87 & -0.01 \\
$Z: 4 / 4$ & 3.17 & 21.96 & 9.23 & -0.87 & -0.02 \\
$Z: 2 / 14$ & 3.13 & 43.49 & 8.13 & -0.98 & -0.05 \\
$Z: 14 / 2$ & 3.20 & 44.38 & 9.34 & -0.76 & -0.04 \\
$Z: 14 / 14$ & 3.17 & 76.81 & -0.87 & -0.04 \\
\hline \hline
\end{tabular}

Even if the lattice mismatch between 2D GaN and 2D AlN is small and only $0.08 \AA$, whether this small lattice mismatch may cause reconstruction at the boundary has been our concern. That composite structures survived MD simulations at temperatures as high as $1000 \mathrm{~K}$ and maintained their honeycomblike structures eliminates the possibility of a boundary reconstruction. We, nonetheless, performed structure optimizations of $Z:(2 / 2)$ using the supercell of $1 \times 2,1 \times$ 4 , and $1 \times 8$ (increasing the size along the interface), whereby constraints hindering a possible reconstruction in the primitive unit cell are removed. After the structure optimizations, no reconstruction was deduced at the boundaries.

Small negative $\bar{E}_{j}$ in Table I suggest that a periodic composite structure is slightly unfavorable relative to the periodic 2D SL GaN and AlN. However, segregation of 2D SL
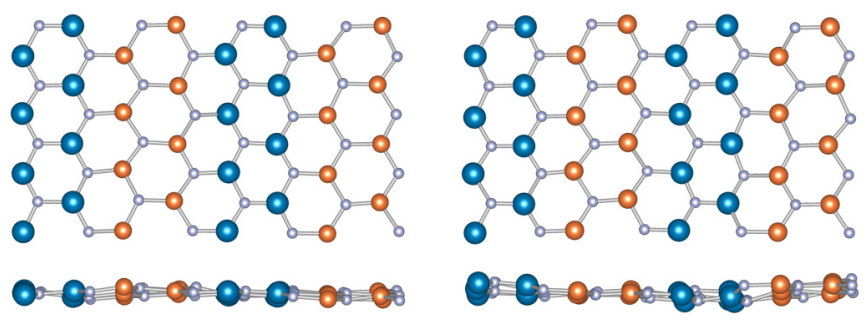

$\mathrm{T}=400 \mathrm{~K}$

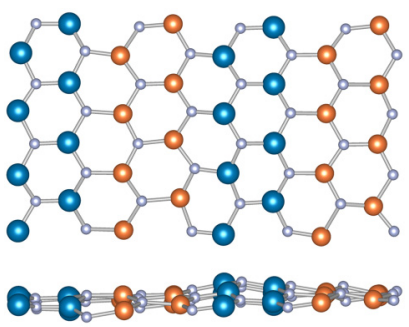

$\mathrm{T}=800 \mathrm{~K}$

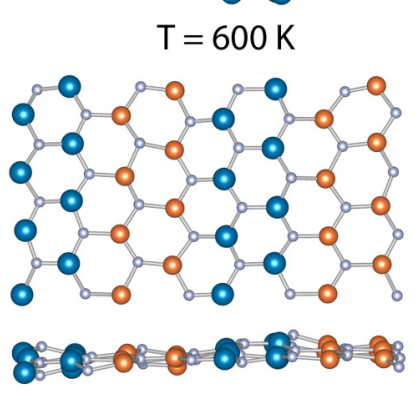

$\mathrm{T}=1000 \mathrm{~K}$

FIG. 3. Snapshots of the atomic configuration of the composite $Z:(p / q)$ obtained from MD simulations at $400,600,800$, and $1000 \mathrm{~K}$. Simulations are performed in the $(2 \times 4)$ supercell. Total simulation time is $4 \mathrm{ps,} \mathrm{including} \mathrm{the} \mathrm{preliminary} T=200 \mathrm{~K}$ step, where the simulation lasts $0.5 \mathrm{ps}$ at each temperature until $800 \mathrm{~K}$, followed by another 2 ps at $1000 \mathrm{~K}$.
$\mathrm{GaN}$ and AlN from a periodic composite cannot be mediated by a small and negative junction formation energy. Moreover, following the growth of $\mathrm{GaN}$ (AlN) stripe, the saturation of $\mathrm{N}$ atoms at the edge by $\mathrm{Al}(\mathrm{Ga})$ atoms and hence the start of the growth of AlN ( $\mathrm{GaN})$ along the zigzag edge can occur without any energy barrier, which is an exothermic process. It is also shown that such a composite can survive high-temperature thermal excitations with uniform boundaries.

\section{B. $(\mathrm{GaN})_{p} /(\mathrm{AIN})_{q}$ composite structures}

Figures 4(a)-4(o) summarize the situation with the composites constructed from very thin stripes of $1 \mathrm{D} \mathrm{GaN}$ and AlN, namely, $Z:(1 / 1), Z:(2 / 2)$, and $Z:(4 / 4) . Z:(1 / 1)$ attains an indirect band gap $E_{g, i}=2.62 \mathrm{eV}$, which is different from its parent constituents, yet $0.08 \mathrm{eV}$ larger than their average value of $2.54 \mathrm{eV}$. Constituents provide practically equal contributions to the state density. The electronic properties of these composites can be tuned by changing the value of $p$ and $q . Z:(2 / 2)$ has an indirect band gap $E_{g, i}=2.57 \mathrm{eV}$, which is also $0.03 \mathrm{eV}$ larger than the average value of the constituents, but closer to it. In Figs. 4(k)-4(o), the composite $Z:(4 / 4)$ has a band structure similar to that of $Z:(2 / 2)$, except it contains more bands and the fundamental band gap is reduced by $0.10 \mathrm{eV}$, becoming smaller than the average band gap of the constituents. Notably, as $p=q$ increases from 2 to 4 , the tendency to the phase separation through confinement of the electronic states to one of the stripes increases. For example, while the states at the edges of the conduction and valence bands of $Z:(2 / 2)$ are not confined, the states at the top of the valence band of $Z:(4 / 4)$ start to be confined to GaN stripe since the top of the valence band of AlN occurs at a lower energy as depicted by the charge density isosurfaces in Fig. 4(o).

\section{C. $\delta$ doping}

The $\delta$ doping, where a very thin stripe of one constituent is implemented in a wide stripe of the other constituent, is another critical case of the composite structure. In Fig. 5(a), the structure $Z:(14 / 2)$ is taken as the model to mimic the $\delta$ doping of $2 \mathrm{D} \mathrm{GaN}$ by a narrow $1 \mathrm{D}$ AlN stripe. While the electronic structure is mainly due to the large GaN stripes, which is similar to that of the 2D parent GaN, a narrow AlN stripe 
(a)



(e)

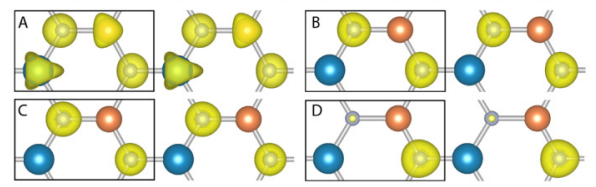

$\mathrm{Z}:(\mathrm{GaN})_{2} /(\mathrm{AIN})_{2}$

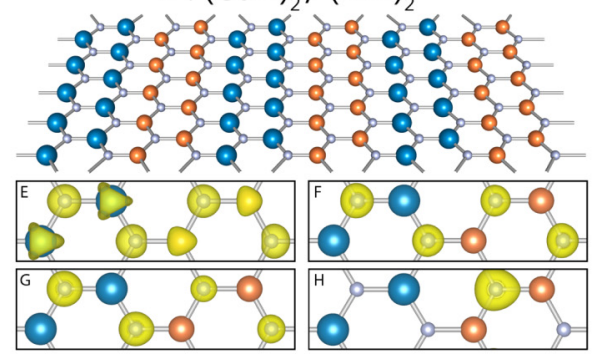

(j)

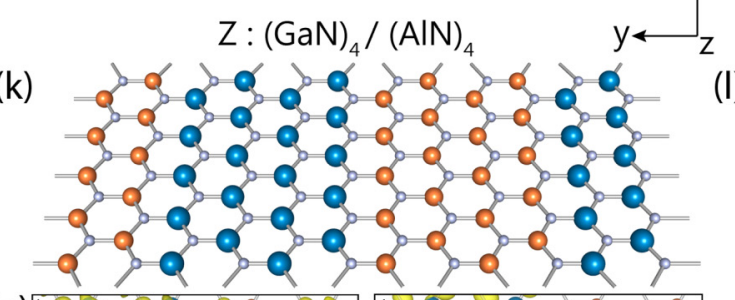

(o)

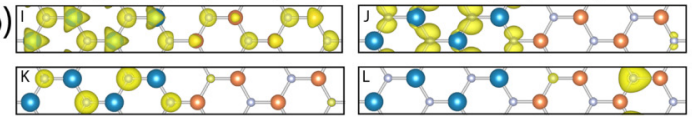

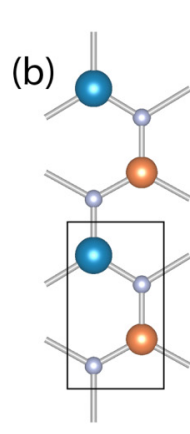
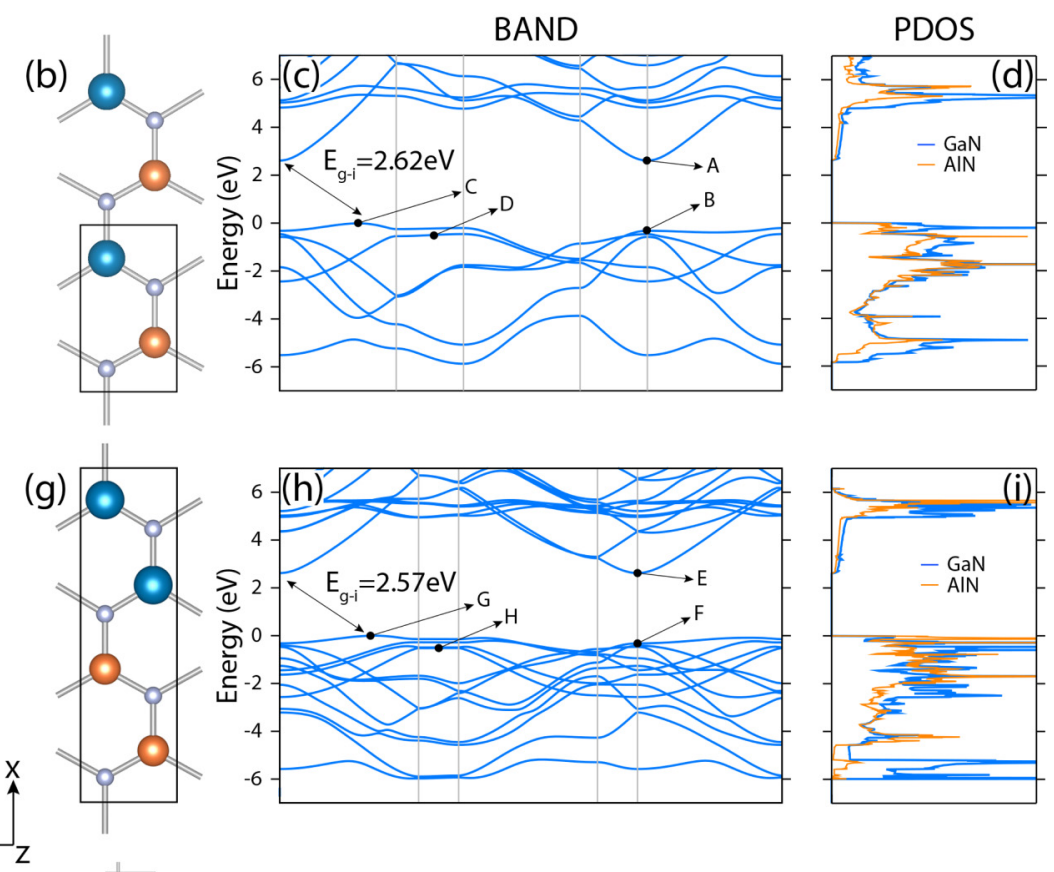

(I)
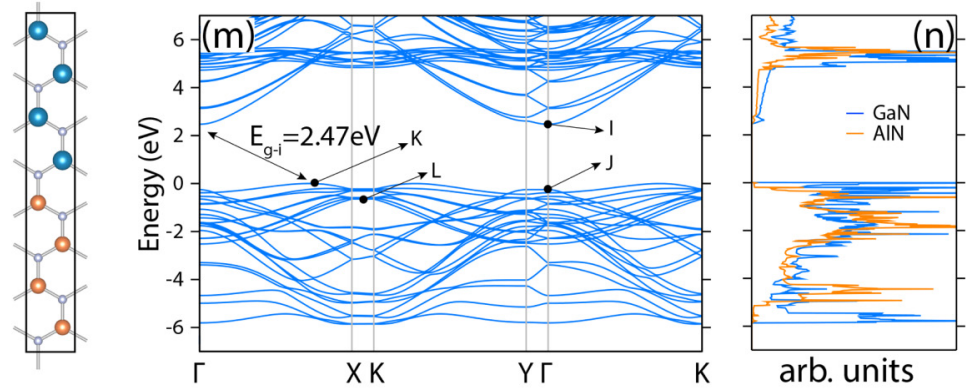

FIG. 4. (a) A perspective view of the composite structure $Z:(\mathrm{GaN})_{1} /(\mathrm{AlN})_{1}$. (b) The unit cell. (c) Energy band structure with the indirect band gap $E_{g, i}$. Zero of energy is set at the top of the valence band. (d) Densities of states projected to GaN and AlN. (e) Charge density isosurfaces of the selected band states $\rho_{n, k}(\mathbf{r})$ indicated as A, B, C, D in (c). (f)-(j) Same for $Z:(\mathrm{GaN})_{2} /(\mathrm{AlN})_{2}$ constructed from the stripes having twice the width of (a). (k)-(o) Same for $Z:(\mathrm{GaN})_{4} /(\mathrm{AlN})_{4}$ constructed from stripes having four times the width of (a). Ga, Al, and $\mathrm{N}$ atoms are shown by large turquoise, medium orange, and small gray balls, respectively.

implemented in $\mathrm{GaN}$ results in an increase of the fundamental band gap from $E_{g, i}=2.16$ to $2.22 \mathrm{eV}$ between the minimum of the conduction band at $\Gamma$ and the maximum of the valence band, near $X$ points. Based on the charge density isosurfaces of states in Fig. 5(c), while narrow AlN stripe contributes to the $\mathrm{GaN}$ states at the minimum of the conduction band, the states at the top of the valence band are constructed from $\mathrm{GaN}$ since the bonding AlN states occur at energies slightly lower than the top of the valence band. In fact, the states indicated by $\mathrm{C}$ in Fig. 5(b) are 1D localized states along $X-K$ direction, which are confined to AlN. Accordingly, the gap widening occurs in the direct space locally throughout the narrow AlN stripe.

In the $\delta$ doping of wide AlN stripe by narrow GaN stripe represented by $Z:(2 / 14)$ in Fig. 5(d), the indirect fundamental gap of parent 2D AlN is reduced to $E_{g, i}=2.70 \mathrm{eV}$ since $\mathrm{GaN}$ has a smaller gap and the bands of the bonding GaN states occurred above the top of the valence band of AlN. Namely, in Fig. 5(e) the (band) states indicated by E and F are derived from $\mathrm{GaN}$ and reduce the fundamental gap of AlN. This situation is depicted by the charge distribution of these 1D states in Fig. 5(f), which are localized in the narrow GaN stripe, but propagating along the $K-X$ direction. Hence, the fundamental band gap of AIN is reduced locally along the GaN stripe.

\section{Multiple quantum well structures and heterostructures}

Since the fundamental band gaps of constituent 2D GaN and AlN are different, the composite structure discussed above offers also the realization of the periodically repeating multiple quantum well structures provided that $p$ and $q$ are sufficiently large as shown in Fig. 6(a). Isosurfaces of the total charge shows Ga-N and Al-N bonds with charge accumulation around more electronegative $\mathrm{N}$ anion sites and two different regions of GaN and AlN stripes. In Fig. 6(b), the distribution of the bond lengths in the primitive unit cell are presented in $\mathrm{GaN}$ and AlN stripes. Due to the minute lattice match, the bonds in $\mathrm{GaN}$ region are smaller than the parent 2D GaN; on the contrary, Al-N bonds in AlN region are larger than those in the parent 2D AlN. Upon formation of the heterostructure, the lattice mismatch is compensated by both constituents, whereby $\mathrm{GaN}$ bonds conform to this situation by squeezing a small 


\section{$\delta$ - DOPING STRUCTURE}

$$
\mathrm{Z}:(\mathrm{GaN})_{14} /(\mathrm{AIN})_{2}
$$

(a)

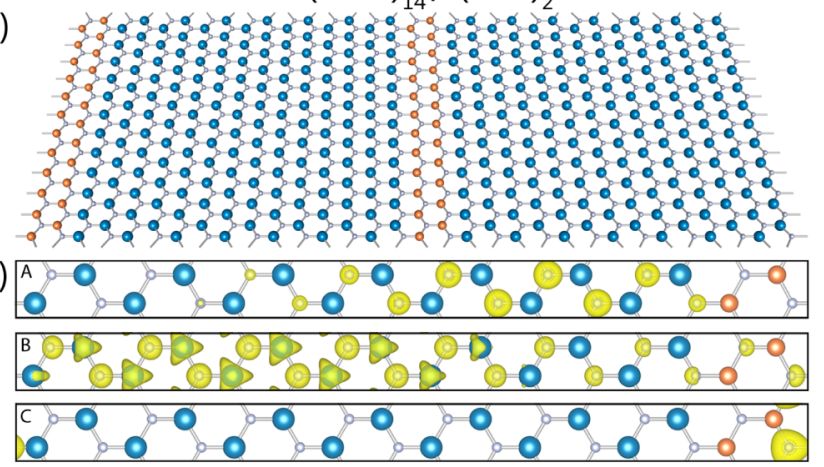

(d)

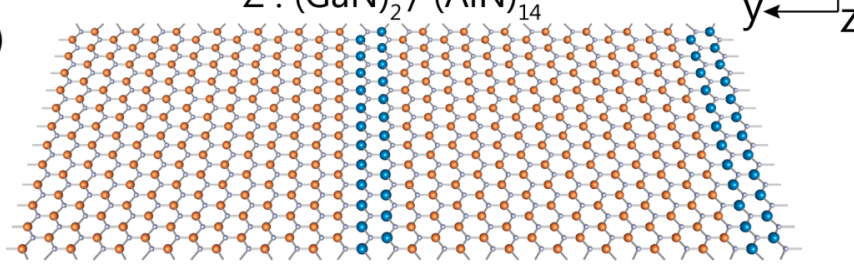

(f)

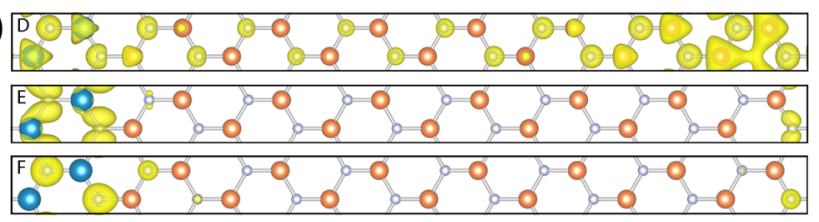

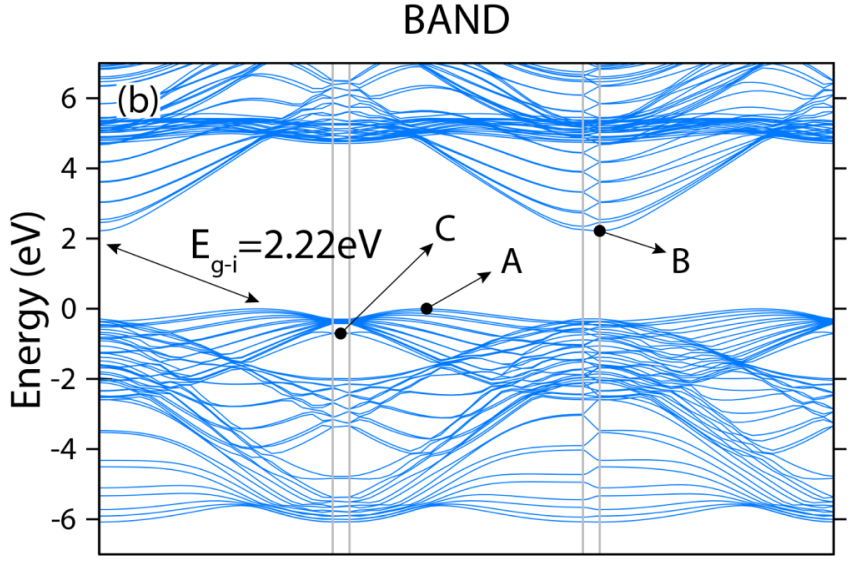

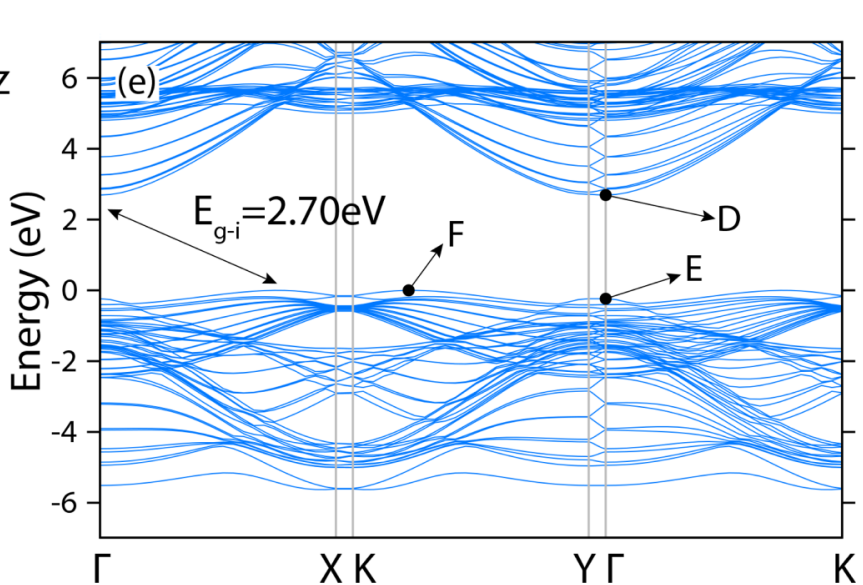

FIG. 5. $\delta$ doping: (a) A perspective view of the composite structure $Z:(14 / 2)$, where wide GaN stripes are $\delta$ doped by very narrow stripes of AlN. (b) Electronic energy bands with the indirect band gap $E_{g, i}$ shown by an arrow. Zero of energy is set at the top of the valence band. (c) Isosurfaces of the charge density of the selected band states indicated in (b). (d)-(f) Same for Z:(2/14).

amount, while AlN bonds expand slightly. Notably, as a result of the breaking of the threefold rotation symmetry due to the boundaries $I_{1}$ and $I_{2}$, two different bonds in either region are distinguished: the lateral bonds along the $y$ axis are relatively longer than the tilted bonds. Additionally, in the center of the stripe, $\mathrm{GaN}$ bonds become gradually shorter, while this situation is reversed in the AlN stripe.

Charge density at each bond in GaN and AlN stripes exhibits small variations around their total valence charge amounting to eight electrons. However, this variation appears to be dramatic at the close proximity of $I_{1}$ and $I_{2}$, indicating charge transfer between the constituent stripes across the boundaries. In Fig. 6(c), the analysis of the charge density [38] shows that the electrons are depleted from the tilted AlN bond to the left of $\mathrm{I}_{2}$, nevertheless, they are accumulated at the $\mathrm{GaN}$ bond to the left of $\mathrm{I}_{1}$ as depicted in the $(x, z)$-plane averaged total charge calculated at the centers of the tilted bonds. This charge transfer affects the electronic potential and fixes the band lineup. In Fig. 6(d), the variation of the $(x, z)$-plane averaged potential energy along the $y$ axis and its average $\bar{V}(y)$ are plotted. Here, we note two crucial features: (i) At the interfaces, the jumps of $\bar{V}(y)$ occur since the average potential energies of parent $2 \mathrm{D} \mathrm{GaN}$ and $\mathrm{AlN}$ are different and have values of -2.55 and $-2.61 \mathrm{eV}$, respectively. At the centers of GaN and AlN stripes, the average potential energies are -3.28 and $-3.69 \mathrm{eV}$, respectively. (ii) Owing to the charge transfers at the boundaries, $\bar{V}(y)$ is not flat, but exhibits a zigzag variation.

Here, the theories developed earlier to set the band edges of 3D heterostructures $[40,41]$ in the direct space are adapted to the in-plane heterostructures and the band diagram of $Z:(14 / 14)$ heterostructure in the direct space is plotted. First, the energies of the minimum of conduction band (CB) and the maximum of the valence band (VB) of $2 \mathrm{D} \mathrm{GaN}(1.42$ and $-0.73 \mathrm{eV})$ and $2 \mathrm{D}$ AlN $(2.33$ and $-0.58 \mathrm{eV})$ relative to their average potential energies are marked by red lines in Fig. 6(d). According to this band diagram, which displays a type-I alignment, the dip of the $\mathrm{CB}$ becomes a quantum well for excess electrons in the $\mathrm{GaN}$ stripe and the hill of $\mathrm{VB}$ at $\mathrm{I}_{1}$ accommodates excess holes also in GaN. Since $\bar{V}(y)$ is slightly tilted, the band gap occurs between the different locations in the indirect space and attains a value smaller than the band gap of $2 \mathrm{D} \mathrm{GaN}$. This controversy will be resolved in the next paragraph.

Next, we used an alternative approach in order to determine the band diagram by calculating the local density of states (LDOS) at the centers of all bonds in the unit cell. The band edges inferred this way are marked with dots in the same figure. The size of the dots is indicative of the uncertainty in setting the energy locations. Some of these LDOSs corresponding to the bonds at the selected locations in the unit cell are shown in Fig. 6(f). Even if the band diagram inferred from 


\section{$\mathrm{Z}:(\mathrm{GaN})_{14} /(\mathrm{AIN})_{14}$ HETEROSTRUCTURE}

(a)
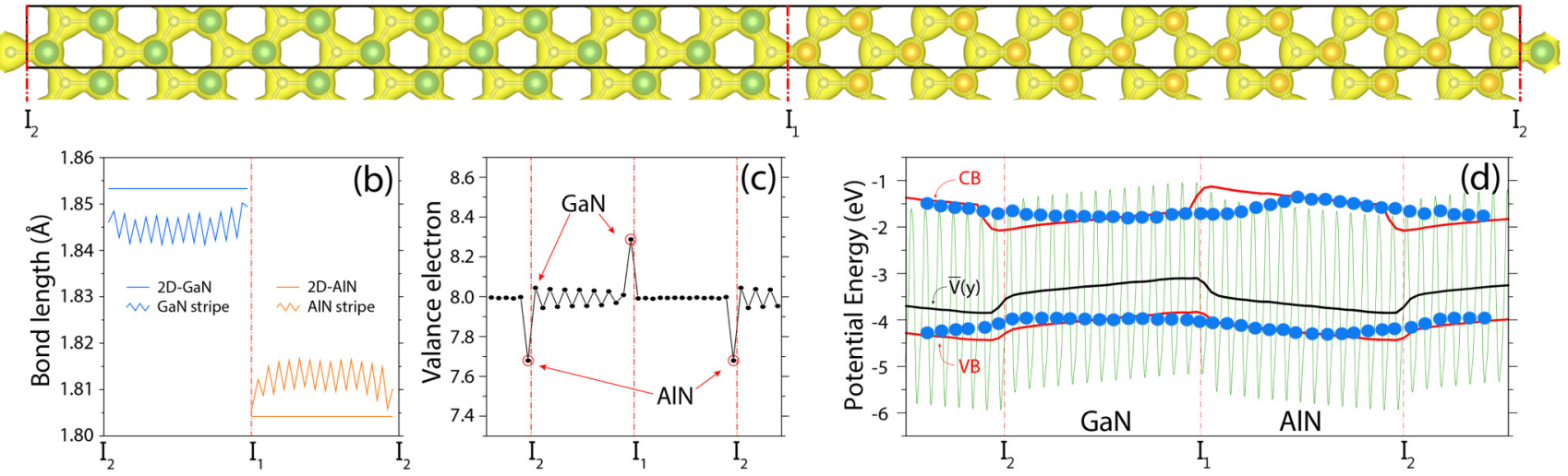

(e)

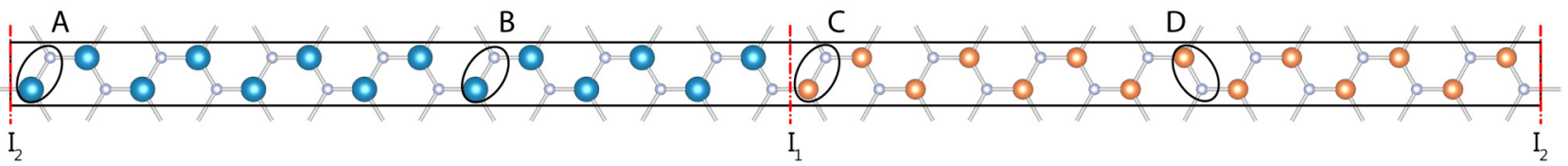

(f)
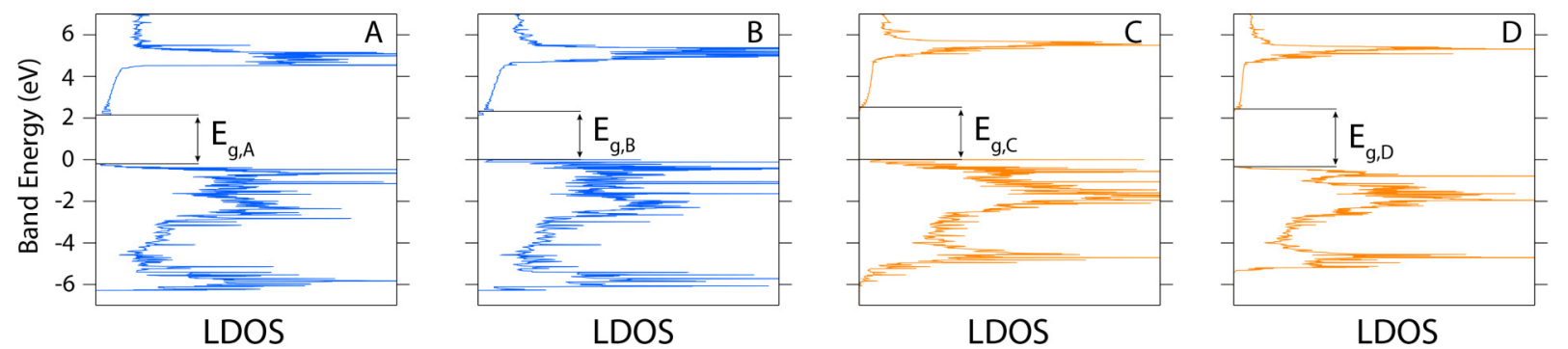

FIG. 6. (a) The unit cell and atomic configuration of the in-plane heterostructure $Z:(\mathrm{GaN})_{14} /(\mathrm{AlN})_{14}$ together with the isosurfaces of the total charge density. $I_{1}$ and $I_{2}$ are boundaries (or interfaces) between stripes. (b) The variation of GaN and AlN bond lengths in the unit cell. Bond lengths of the parent 2D GaN and AlN constituents are also indicated. (c) The total charge calculated at the centers of the tilted bonds in (a) shows charge transfer at the boundaries. (d) Variation of the $(x, z)$-plane averaged potential energy (green lines) and its average $\bar{V}(y)$ (black line). The minimum of the conduction band CB and the maximum of the valence band VB (red line) along the $y$ direction is plotted. On the same plot, $\mathrm{CB}$ and VB inferred from LDOS at the bond centers are also marked by blue dots. The band edges in the direct space display a multiple quantum well structure. (e) Specific GaN and AlN bonds in the unit cell are indicated by A, B, C, D. (f) LDOSs corresponding to the bonds A, B, C, D.

LDOS attains a multiple quantum well structure, it exhibits differences from that obtained using the theories developed for 3D heterostructures [40,41]. First of all, some of the dots indicating the minimum conduction band and maximum valence band states in fact correspond to the confined states; because of confinement effect, they occur at higher energies for the conduction band and at lower energies for the valence band. Accordingly, the effects of the tilting of $\bar{V}(y)$ are weakened and the quantum well structure appear to vary rather smoothly with the band discontinuities at the boundaries disappearing. Also, the minimum gap in the band diagram inferred from LDOS calculations coincides with the minimum gap of $2.16 \mathrm{eV}$ calculated in the momentum space since they are based on the result of the same calculation. Normally, the gap is expected to be slightly larger due to the confinement effect; yet, it decreases related with the tilting of the band edges and attains the value of the band gap calculated for 2D GaN coincidentally.

The band structure of $Z:(14 / 14)$ in momentum space is presented in Fig. 7. The isosurface charge densities of specific states at the edges of the valence and conduction bands are presented also in the same figure. The states $\mathrm{A}, \mathrm{B}$, and C at the top of the valence band are confined to the GaN stripe since they cannot find partners in AIN to match their momentum and energy. They are flat and dispersionless along $X-K$ and $Y-\Gamma$ directions of the BZ (perpendicular to the stripes), but propagate along the GaN stripe. The unoccupied conduction band states $\mathrm{D}$ and $\mathrm{E}$ are also confined to the $\mathrm{GaN}$ stripe. These states can be occupied by the excess electrons, such as electrons of the ionized donors placed in the AlN stripe through the modulation doping. Confinements of the states at the edges of both conduction and valence bands of $\mathrm{GaN}$ demonstrate that the heterostructure has a type-I band alignment in compliance with Fig. 7. The state $\mathrm{E}$ has higher energy and is confined at the AlN site since it cannot find any matching state in the GaN stripe to propagate throughout the heterostructure. However, specific states in the band continua can propagate throughout the stripes along the $y$ direction when their momentum and energy are matched in the adjacent stripes.

\section{E. Finite-size AIN/GaN/AIN junctions}

We now present our results of finite-size $(\mathrm{AlN})_{13} /(\mathrm{GaN})_{13} /$ $(\mathrm{AlN})_{13}$ in-plane junction constructed by AlN, GaN, and AlN 


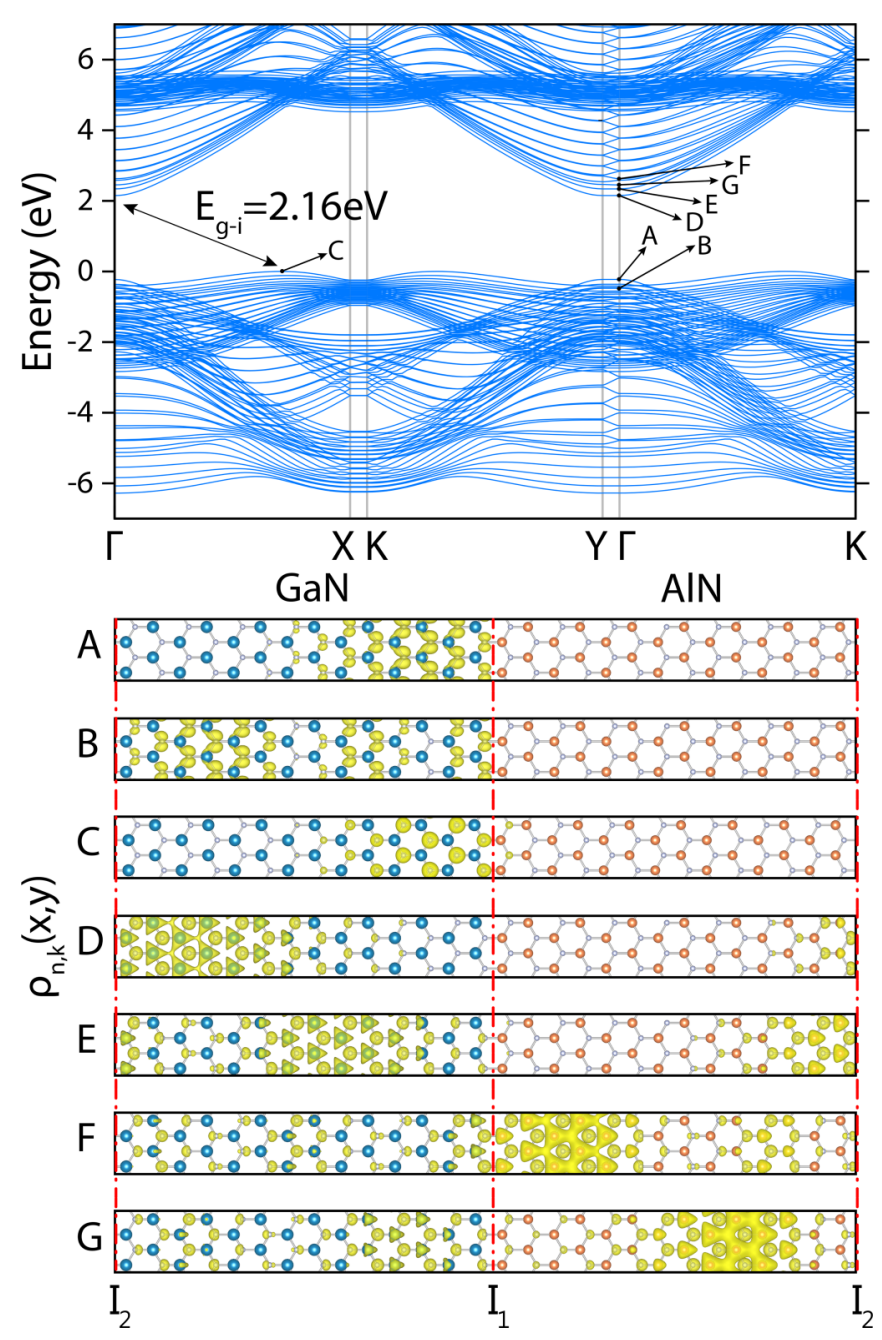

FIG. 7. Energy band structure of $Z:(\mathrm{GaN})_{14} /(\mathrm{AlN})_{14}$ in momentum space. The isosurface charge densities of the band states $\mathrm{A}, \mathrm{B}$, ..., $\mathrm{G}$ presented in a plane comprising two adjacent unit cells with $\mathrm{I}_{1}$ and $I_{2}$ boundaries demonstrate the confinement of specific states in different stripes and multiple quantum well formation.

stripes, which are commensurately joined along their zigzag edges and capped by a vacuum space of $24 \AA$ from both ends as described in Fig. 8(a). Dangling bonds at both edges of the ribbon oozing from low coordinated $\mathrm{N}$ and $\mathrm{Al}$ atoms are saturated by hydrogen atoms. This way, spurious localized states due to those low coordinated edge atoms are discarded from the band gap. Differences between the two edges of the heterostructure give rise to an asymmetry in its physical properties. The $(x, z)$-plane averaged potential and its average value $\bar{V}(y)$ in Fig. 8(b) display these asymmetries. Having obtained $\bar{V}(y)$, we next determine $\mathrm{CB}$ and VB in the direct space. To this end, we use the energies of the band edges of 2D GaN and 2D AlN calculated with respect to their average potentials and mark them relative to $\bar{V}(y)$ in Fig. 8(b). In accordance with the effective mass approximation [42], the $\mathrm{CB}$ and VB band edges determined this way form the quantum well structure in $\mathrm{GaN}$ stripe and have expectantly sharp discontinuities at the boundaries. Alternatively, the minimum of the conduction band and the maximum of the valence band (a)

\section{$\mathrm{Z}:(\mathrm{AIN})_{13} /(\mathrm{GaN})_{13} /(\mathrm{AIN})_{13}$ HETEROSTRUCTURE}
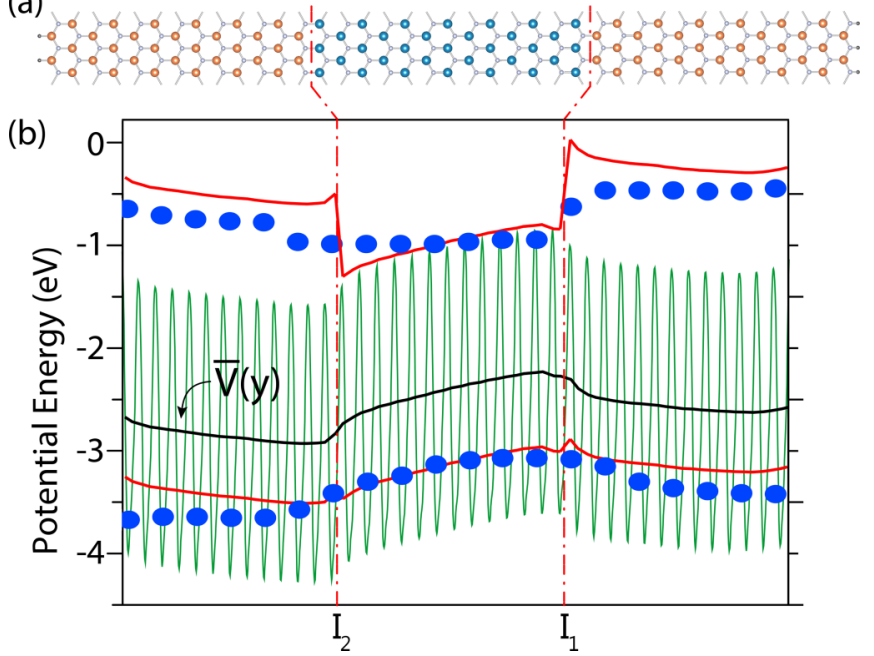

FIG. 8. Finite-size $(\mathrm{AlN})_{13} /(\mathrm{GaN})_{13} /(\mathrm{AlN})_{13}$ junction forming a quantum well structure. (a) The atomic structure of AlN, GaN, and AlN stripes capped by a vacuum space of $24 \AA$ from both sides. The boundaries between the stripes are $\mathrm{I}_{1}$ and $\mathrm{I}_{2}$. Twofold-coordinated $\mathrm{Al}$ and and $\mathrm{N}$ atoms of the two edges are saturated by $\mathrm{H}$ atoms. (b) $(x, z)$-plane averaged electronic potential and its average $\bar{V}(y)$. The $\mathrm{CB}$ and $\mathrm{VB}$ edges of the bands are inferred relative to $\bar{V}(y)$ as explained in the text and shown by red lines. The states at the maximum of the valence and those at the minimum of the conduction band are retrieved from LDOSs calculated at the center of the bonds in the unit cell and are marked by dots. The size of the dots is indicative of the uncertainty in determining the energies of these states. Ga, Al, and $\mathrm{N}$ atoms are represented by large turquoise, medium orange, and small gray balls, respectively.

states are retrieved from the band edges of LDOSs and marked as dots in Fig. 8(b). Some of these states in the dip of the quantum well near $\mathrm{I}_{2}$ and those at the hill near $\mathrm{I}_{1}$ are actually confined states and make the apparent band gap in the quantum well wider. Moreover, the discontinuities and the tilting of $\mathrm{CB}$ and VB are smoothened. The AlN stripe, which plays the role of the barrier due to its relatively larger band gap, can also allow the confinement of some states, which cannot find a matching partner in the adjacent GaN stripe. Such a possibility was pointed out earlier for bulk ALGAS heterostructures [43]. The band structure of the junction in the direct space is presented in Fig. 8(b).

\section{F. Patterned GaN/AIN core/shell structures}

We note that the dimensionality of the electronic states of $Z:(p / q)$ composite structures constructed from $\mathrm{GaN}$ and $\mathrm{AlN}$ stripes is reduced from $2 \mathrm{D}$ to $1 \mathrm{D}$ as a result of the confinements and hence localization of the states to different stripes for specific $p$ and $q$ values. The dimensionality can be reduced further from 1D to $0 \mathrm{D}$, if one constructs a GaN/AlN core/shell structure or its periodically repeating array [44]. In Fig. 9, we present such a patterned array of GaN/AlN core/shell, where SL $\mathrm{GaN}$ zones of $(5 \times 5)$ squares are implemented commensurately into 2D SL AlN to form a mesh with the supercell of $(12 \times 12)$. The calculated electronic structure of this mesh corresponding to the optimized atomic structure 


\section{ARRAY OF GaN/AIN CORE/SHELL}

(a)
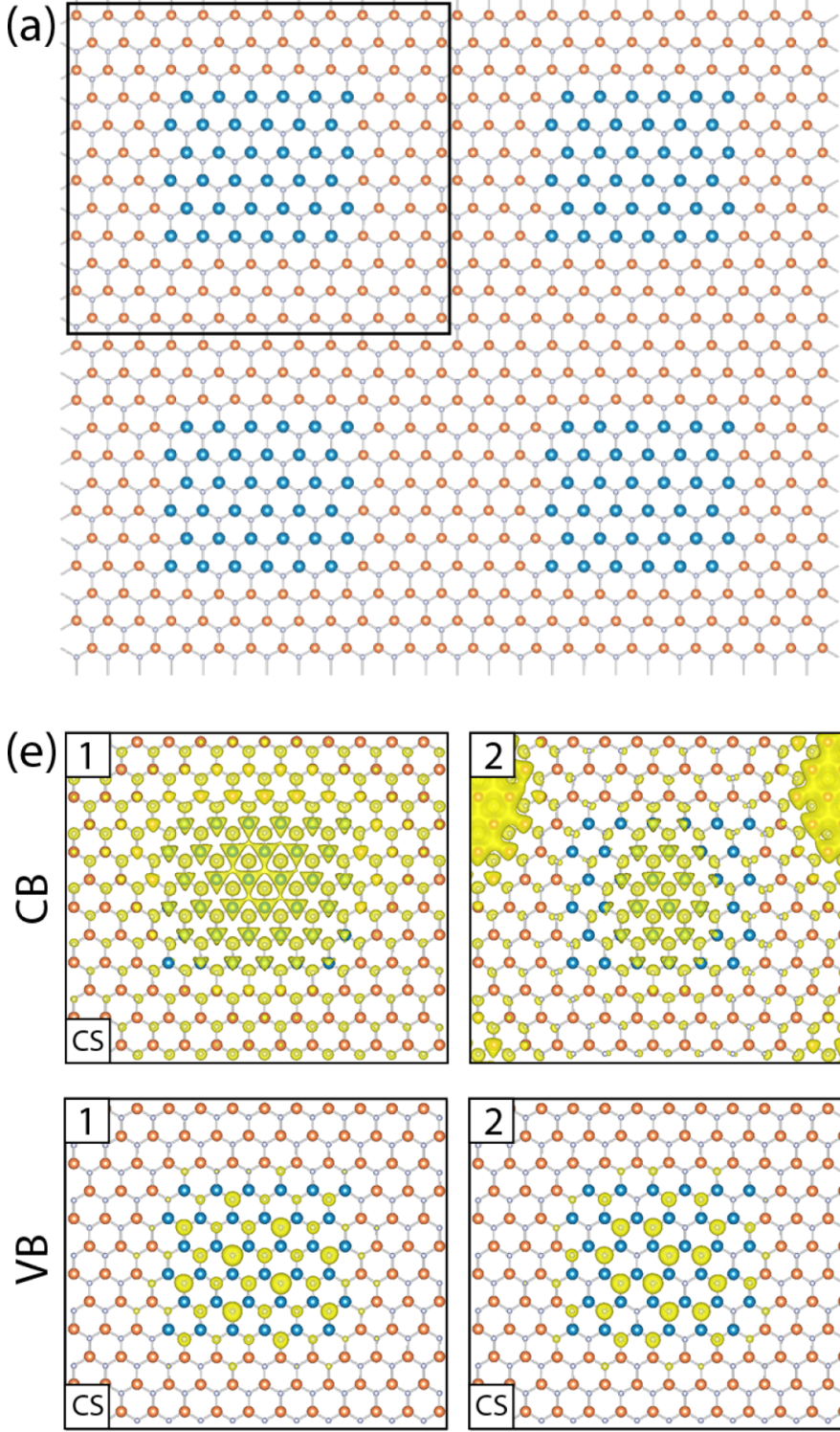

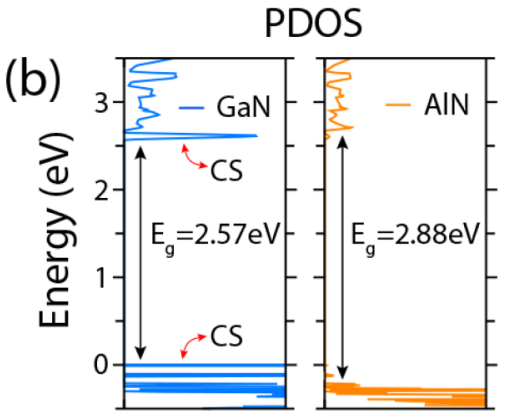

arb. units

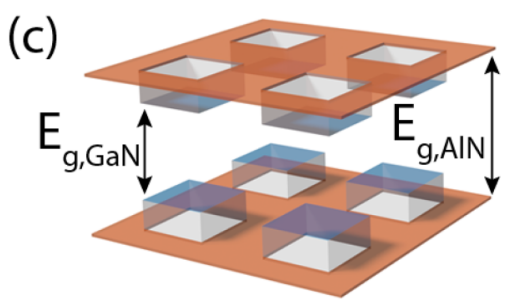

BAND

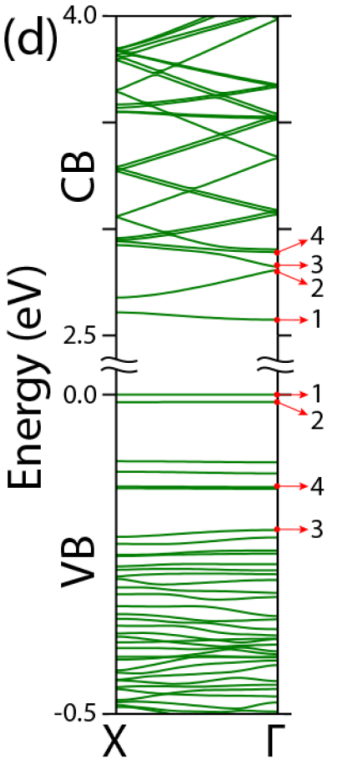

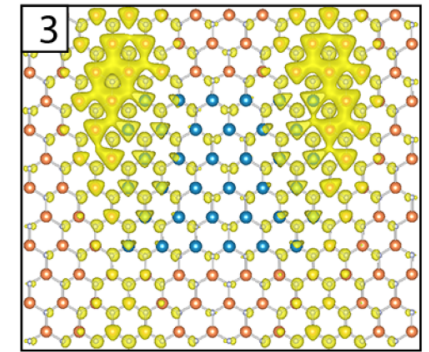

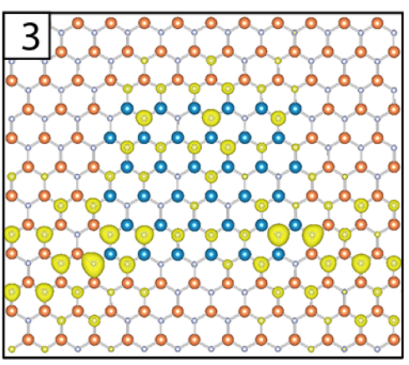

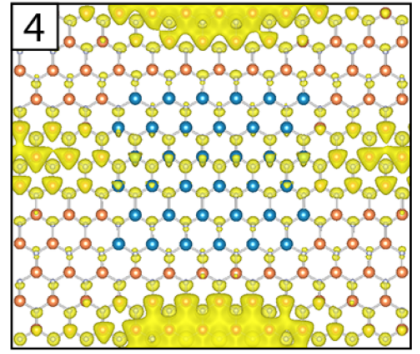

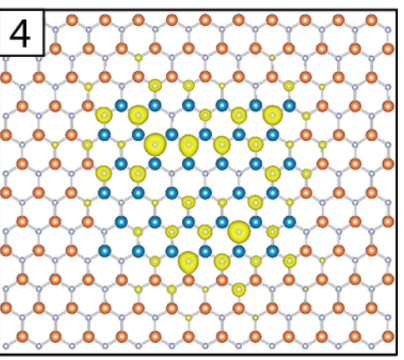

FIG. 9. (a) Atomic configuration of square SL GaN zones joined commensurately to SL AlN forming a regular patterned GaN/AlN core/shell array. $\mathrm{Ga}, \mathrm{Al}$, and $\mathrm{N}$ atoms are shown by large turquoise, medium orange, and small gray balls, respectively. The supercell corresponding to a single GaN/AlN core/shell structure is delineated. (b) LDOSs at the centers of GaN core and AlN shell. Sharp peaks at the band edges are due to confined states (CS). (c) Schematic description of the band-gap landscape in the $(x, y)$ plane showing 0D wells for the valence and conduction band states of $\mathrm{GaN}$ forming a quantum dot. (d) Energy bands near the gap region. Flat (dispersionless) bands with numerals correspond to confined states (or quantum dot states). The states $2-4$ in the conduction band are slightly delocalized. (e) Charge density isosurfaces of selected confined and itinerant states specified by numerals in (d).

shows a planar landscape of the band gap, which shall be $2.16 \mathrm{eV}$ in $\mathrm{GaN}$ core, but relatively larger and $2.91 \mathrm{eV}$ in AlN shell. Each GaN core behaves as if it is a quantum dot with confined electronic states. However, states confined to the cores or quantum dots have energy above the conduction band and below the valence band edge. The smaller the size of the core, the higher (lower) are the confined states from the conduction (valence) band edges. In Fig. 9(b), the sharp peaks near the band edges of GaN core are due to the confined states and make the band gap appear as than $2.57 \mathrm{eV}$. The peaks originating from the confined states disappear in the AlN shell. The level spacings and the ordering of the confined states can be monitored by the size and geometric shape of the cores. The states of AlN shell around GaN core propagate in the multiply connected regions with wave vectors $\left(k_{x}, k_{y}\right)$ and display the band structure of a 2D supercrystal. This way, one can generate a 2D artificial crystal with a desired energy spectrum and fundamental band gap.

Figure 9(c) presents a schematic description of the band-gap landscape in the $(x, y)$ plane showing $0 \mathrm{D}$ wells for the valence and conduction band states of $\mathrm{GaN}$ forming an array of quantum dots. In Fig. 9(d), the energy band structure of the patterned array is shown. Nearly flat (dispersionless) bands near the band edges are confined states, which lead to sharp 
peaks at the band edges. In Fig. 9(e), we plot the isosurfaces of the states, which are identified by the numerals. Seven states at the top of the valence band of the GaN core are strongly confined. Because of the pseudopotential used in our calculation, charge appears as localized around the $\mathrm{N}$ atoms. In the conduction band, only one strongly confined state is identified. Higher-lying states are delocalized and appear as itinerant AIN states in the shell region. The envelope functions of the states confined at the top of the valence band can be obtained within the effective mass theory [42]. For a square core with a finite potential depth, these states can be represented in terms of $\sin (\xi x)$ and $\sin (\xi y)$ like functions. For circular cores, the envelope functions can be expressed in terms of the cylindrical Bessel functions.

Conversely, if the size of GaN cores is reduced and the AlN shell is narrowed down, the confined electronic states start to be delocalized, yet become propagating in $2 \mathrm{D}$, attributing novel features to the resulting composite material. Alternatively, periodic domains with different properties can be realized by the patterned adsorption adatoms such as $\mathrm{H}$ to host 2D SL GaN or AlN. This way, magnetic domains forming a mesh structure can be realized [45].

\section{G. Effect of substrate}

The above discussions related to the structural and electronic properties of composites are pertinent to the freestanding (suspended) single layers. However, SL GaN and AlN crystals do not exist in nature, single-layer GaN and AlN, as well as their composites, cannot be produced from their parent 3D crystals through exfoliation or laser ablation. Hence, the growth of GaN/AlN composites on suitable substrates appears to be the only way to produce composite structures. Once grown on a substrate, the strength of the interaction between the grown composite and substrate becomes crucial. If the interaction is significant, the physical properties predicted for the suspended single layers may undergo dramatic modifications. We therefore investigate the substrate-composite interaction and possible induced changes in the physical properties. Earlier, a 2D single-layer GaN overlayer placed on metallic $\mathrm{Al}(111)$ surface and semiconducting blue phosphorene was investigated [25]. The spacings between the GaN overlayer and two different substrates, i.e., $\mathrm{Al}(111)$ slab and blue phosphorene, were found to be 3.17 and $2.97 \AA$, respectively. When compared to the sums of the atomic radii, these separations are rather large and prevent the overlayer from setting up significant interaction with substrates. In fact, the analysis of the density of states projected onto the overlayer resulted in an electronic structure similar to that of the free-standing 2D GaN, indicating minute substrate-GaN interaction. In particular, the interaction between 2D GaN overlayer and blue phosphorene substrate was negligible. We found similar results for the 2D single-layer AlN grown on $\mathrm{Al}(111)$ and blue phosphorene substrates [46].

Here, we placed a composite structure $(\mathrm{GaN})_{2} /(\mathrm{AlN})_{2}$ on an $\mathrm{Al}(111)$ slab and phosphorene, then optimized the atomic structure in order to determine the equilibrium geometry. Since $\mathrm{Al}(111)$ is not lattice matched to the composite structure, we expanded it to ensure the lattice matching and to use the translational periodicity. We note that by expanding $\mathrm{Al}(111)$, we enhanced its reactivity to the overlayer, hence created even

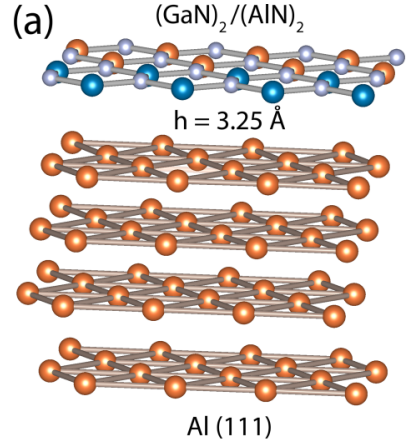

(b)

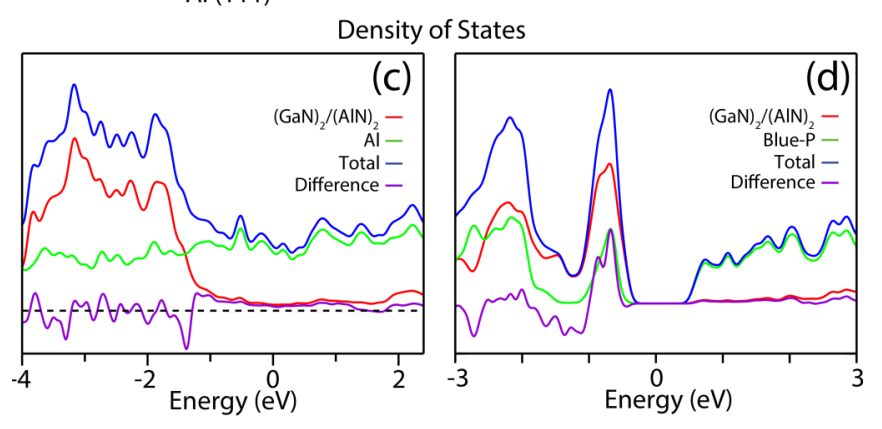

FIG. 10. Effect of substrate on the in-plane composite structures: (a) optimized structure of $(\mathrm{GaN})_{2} /(\mathrm{AlN})_{2}$ placed on a four-layer $\mathrm{Al}(111)$ slab; (b) $(\mathrm{GaN})_{2} /(\mathrm{AlN})_{2}$ placed on a SL blue phosphorene; (c) local density of states (LDOS) on the composite structure and aluminum substrate, and total density of states. The difference between LDOS on the overlayer $(\mathrm{GaN})_{2} /(\mathrm{AlN})_{2}$ and free-standing $(\mathrm{GaN})_{2} /(\mathrm{AlN})_{2}$ of Fig. 4 (f) is shown by purple lines. (d) LDOSs, total and difference densities of states similar to (c).

more severe conditions for the overlayer-substrate interaction. In Figs. 10(a) and 10(b), we show the optimized atomic structure of $Z:(p / q)$ overlayer on $\mathrm{Al}(111)$ slab and latticematched phosphorene. The spacing between composite and $\mathrm{Al}(111)$ is found to be $3.25 \AA$ and that between composite and blue phosphorene $2.77 \AA$. These spacings are large enough to hinder any significant overlayer-substrate interaction. In order to quantify the effect of substrate on the electronic structure, we also calculated LDOSs at the overlayer, at the substrate, and the difference between LDOS and TDOS of bare SL composite in Fig. 4(f). The total, projected, and difference densities of states calculated for the optimized structures presented in Figs. 10(c) and 10(d) indicate the minute effects of the substrates. Within the fundamental band gap of the overlayer, the density of states is negligible. The difference density of states has small fluctuation due to the definition of LDOS and numerical accuracy. Notably, the states near the edge of the valence band occur due to the interaction of phosphorene substrate with the overlayer. However, such an interaction is absent for $\mathrm{Al}(111)$ substrate. These results confirm that significant substrate-composite overlayer interaction to modify the electronic structure of the suspended overlayer can be avoided on specific substrates such as $\mathrm{Al}(111)$ surface.

\section{CONCLUSIONS}

In conclusion, in-plane and commensurate composite structures of single-layer $Z:(\mathrm{GaN})_{p} /(\mathrm{AlN})_{q}$ formed from their 
quasi-1D stripes or 0D GaN cores/AIN shells offer a class of 2D materials showing a diversity of the electronic properties and functionalities. These properties can be tuned by the width of the stripes and geometry of core/shells, as well as their periodic or aperiodic arrangements. While the composite structures constructed from the quasi-1D narrow stripes or small core/shells display properties different from those of the 2D constituents, electronic states start to confine to specific zones as stripes are widened or core/shells grow in size. Under these circumstances, in $Z:(\mathrm{GaN})_{p} /(\mathrm{AlN})_{q}$ heterostructures, 2D states transform to $1 \mathrm{D}$ after confinement, where the energy band diagrams exhibit a multiple quantum well structure in the direct space as a result of the type-I band alignment. However, the character of the type-I band alignment depends on whether the structure is periodic or has a finite size. The $\delta$ doping brings about another interesting feature, whereby the band gap can acquire a local widening or narrowing in the direct space in the direction perpendicular to the boundaries.

When placed (or grown) on specific substrates, the properties predicted for free-standing single-layer heterostructures are shown to be unaffected. Therefore, several properties discussed above can be exploited for a device made by the overlayer of a composite on a suitable substrate.

In the modulation doping of $Z:(\mathrm{GaN})_{p} /(\mathrm{AlN})_{q}$, where the ionized impurities are hosted by AlN, the electrons are confined to GaN stripes, hence, high mobility can be attained by the reduced impurity scattering. Similarly, the patterned array of GaN/AIN core/shell structure behaves like a supercrystal leading to a landscape of the band gap in 2D. Such a supercrystal can be exploited as a photonic band-gap material in 2D. When magnetized through hydrogenation or decoration of specific transition-metal adatoms, the composite structures acquire magnetic properties, which are crucial for spintronics.

\section{ACKNOWLEDGMENTS}

The computational resources are provided by TUBITAK ULAKBIM, High Performance and Grid Computing Center (TR-Grid e-Infrastructure), and the National Center for High Performance Computing of Turkey (UHeM) under Grant No. 5003622015. This work was supported by the Scientific and Technological Research Council of Turkey (TUBITAK) under Project No. 115F088. E.D. acknowledges the financial support from the Turkish Academy of Sciences within Outstanding Young Scientists Award Program (TUBA-GEBIP). S.C. acknowledges financial support from the Turkish Academy of Sciences (TUBA).
[1] L. Esaki and R. Tsu, IBM J. Res. Dev. 14, 61 (1970).

[2] H. Kromer, Proc. IEEE 70, 13 (1982).

[3] K. Von Klitzing, Rev. Mod. Phys. 58, 519 (1986).

[4] D. C. Tsui, H. L. Stormer, and A. C. Gossard, Phys. Rev. Lett. 48, 1559 (1982).

[5] E. E. Mendez, W. I. Wang, B. Ricco, and L. Esaki, Appl. Phys. Lett. 47, 415 (1985).

[6] Y. Cao and D. Jena, Appl. Phys. Lett. 90, 182112 (2007).

[7] H. Li, P. Li, J.-K. Huang, M.-Y. Li, C.-W. Yang, Y. Shi, X.-X. Zhang, and L.-J. Li, ACS Nano 10, 10516 (2016).

[8] X. Duan, C. Wang, J. C. Shaw, R. Cheng, Y. Chen, H. Li, X. Wu, Y. Tang, Q. Zhang, A. Pan, J. Jiang, R. Yu, Y. Huang, and X. Duan, Nat. Nanotechnol. 9, 1024 (2014).

[9] C. Huang, S. Wu, A. M. Sanchez, J. Peters, J. P. Jonathan, R. Beanland, J. S. Ross, P. Rivera, W. Yao, D. H. Cobden, and X. Xu, Nat. Mater. 13, 1096 (2014).

[10] H. Sevincli, M. Topsakal, and S. Ciraci, Phys. Rev. B 78, 245402 (2008).

[11] H. Sahin, S. Cahangirov, M. Topsakal, E. Bekaroglu, E. Akturk, R. T. Senger, and S. Ciraci, Phys. Rev. B 80, 155453 (2009).

[12] M. Topsakal, H. Sevincli, and S. Ciraci, Appl. Phys. Lett. 92, 173118 (2008).

[13] G.-X. Chen, X.-G. Li, Y.-P. Wang, J. N. Fry, and H.-P. Cheng, Phys. Rev. B 95, 045302 (2017).

[14] S. Cahangirov and S. Ciraci, Phys. Rev. B 80, 075305 (2009).

[15] O. Gulseren, T. Yildirim, and S. Ciraci, Phys. Rev. B 66, 121401(R) (2002).

[16] L. Ci, L. Song, C. Jin, D. Jariwala, D. Wu, Y. Li, A. Srivastava, Z. F. Wang, K. Storr, and L. Balicas, Nat. Mater. 9, 430 (2010).

[17] M. P. Levendorf, C.-J. Kim, L. Brown, P. Y. Huang, R. W. Havener, D. A. Muller, and J. Park, Nature (London) 488, 627 (2012).
[18] Z. Liu, L. Ma, G. Shi, W. Zhou, Y. Gong, S. Lei, X. Yang, J. Zhang, J. Yu, and K. P. Hackenberg, Nat. Nanotechnol. 8, 119 (2013).

[19] H. Lim, S. I. Yong, G. Kim, A.-R. Jang, and H. S. Shin, Chem. Mater. 26, 4891 (2014).

[20] R. Drost, A. Uppstu, F. Schulz, S. K. Hamalainen, M. Ervasti, A. Harju, and P. Liljeroth, Nano Lett. 14, 5128 (2014).

[21] Y. Gong, J. Lin, X. Wang, G. Shi, S. Lei, Z. Lin, X. Zou, G. Ye, R. Vajtai, and B. I. Yakobson, Nat. Mater. 13, 1135 (2014).

[22] V. O. Ozcelik, E. Durgun, and S. Ciraci, J. Phys. Chem. C 119, 13248 (2015).

[23] V. O. Ozcelik and S. Ciraci, Phys. Rev. B 91, 195445 (2015).

[24] E. Durgun, S. Tongay, and S. Ciraci, Phys. Rev. B 72, 075420 (2005).

[25] A. Onen, D. Kecik, E. Durgun, and S. Ciraci, Phys. Rev. B 93, 085431 (2016).

[26] C. Bacaksiz, H. Sahin, H. D. Ozaydin, S. Horzum, R. T. Senger, and F. M. Peeters, Phys. Rev. B 91, 085430 (2015).

[27] D. Kecik, C. Bacaksiz, R. T. Senger, and E. Durgun, Phys. Rev. B 92, 165408 (2015).

[28] P. Tsipas, S. Kassavetis, D. Tsoutsou, E. Xenogiannopoulou, E. Golias, S. A. Giamini, C. Grazianetti, D. Chiappe, A. Molle, and M. Fanciulli, Appl. Phys. Lett. 103, 251605 (2013).

[29] Z. Y. Al Balushi, Y. Zakaria et al., Nat. Mater. 15, 1166 (2016).

[30] P. E. Blöchl, Phys. Rev. B 50, 17953 (1994).

[31] M. Gajdoš, K. Hummer, G. Kresse, J. Furthmüller, and F. Bechstedt, Phys. Rev. B 73, 045112 (2006).

[32] G. Kresse and J. Hafner, Phys. Rev. B 49, 14251 (1994).

[33] G. Kresse and J. Furthmüller, Comput. Mater. Sci. 6, 15 (1996).

[34] J. P. Perdew, K. Burke, and M. Ernzerhof, Phys. Rev. Lett. 77, 3865 (1996). 
[35] S. J. Grimme, Comput. Chem. 27, 1787 (2006).

[36] T. Bucko, J. Hafner, S. Lebegue, and J. G. Angyan, J. Phys. Chem. A 114, 11814 (2010).

[37] H. J. Monkhorst and J. D. Pack, Phys. Rev. B 13, 5188 (1976).

[38] E. Sanville, S. D. Kenny, R. Smith, and G. Henkelman, J. Comput. Chem. 28, 899 (2007).

[39] C. Attaccalite, A. Nguer, E. Canuccia, and M. Grunning, Phys. Chem. Chem. Phys. 17, 9533 (2015).

[40] R. G. Dandrea, C. B. Duke, and A. Zunger, J. Vac. Sci. Technol. B 10, 1744 (1992).
[41] R. G. Dandrea and C. B. Duke, J. Vac. Sci. Technol. B 11, 848 (1993).

[42] W. Kohn and J. M. Luttinger, Phys. Rev. 97, 1721 (1955); 98, 915 (1955).

[43] S. Ciraci and I. P. Batra, Phys. Rev. B 36, 1225 (1987); 38, 1835 (1988).

[44] S. Cahangirov and S. Ciraci, Phys. Rev. B 83, 165448 (2011).

[45] H. Sahin, C. Ataca, and S. Ciraci, Appl. Phys. Lett. 95, 222510 (2009).

[46] D. Kecik, A. Onen, M. Konuk, F. Ersan, E. Durgun, E. Akturk, and S. Ciraci (unpublished). 